\title{
ÉTHIQUE VÉTÉRINAIRE ET SÉLECTION DU CHIEN DE RACE
}

\author{
VETERINARY ETHICS AND PUREBRED DOG SELECTION
}

Par Gilles CHAUDIEU ${ }^{1}$

(Communication présentée le 29 avril 2021, manuscrit accepté le 25 septembre 2021)

\section{RÉSUMÉ}

La cohabitation du chien et de l'Homme s'est traduite par l'émergence d'un morphotype canin ancestral, puis de races primaires, à partir desquelles la sélection a créé les races modernes que nous connaissons actuellement. Les standards en définissent les phénotypes, mais leur interprétation peut privilégier la production de certains morphotypes raciaux déviants, inductrice d'un appauvrissement de la variabilité génétique par pression sélective inappropriée. Le vétérinaire a toute légitimité pour s'impliquer dans le maintien de la nécessaire diversité génétique des effectifs ; il a le devoir de veiller au bien-être et à la santé du chien de race, en rappelant qu'une dépendance interspécifique harmonieuse du chien et de l'Homme impose à ce dernier de pratiquer une sélection morphologique raisonnable et de favoriser l'épanouissement des aptitudes comportementales naturelles dans chaque groupe de races.

Mots-clés : chien, éthique, race, sélection, standard de race.

\begin{abstract}
-ABSTRACT
Relationships between canine and humans have induced the emergence of an ancestral morphotype, then of primary canine breeds, from which the process of selection has created the modern breeds, as they exist actually. Breed standards have established breed phenotypes but their interpretation may facilitate the development of deviating types, from which a loss of genetic variability may result by breeding, as a consequence of an inappropriate selective pressure. The veterinarian is well-founded to contribute in keeping the necessary genetic diversity in populations of dogs; he must take care of welfare and health in purebred dogs, keeping in mind that an harmonious interspecific dependency between dogs and humans enforce a necessary reasonable morphological breeding selection, as well as a broadening of natural behavioural abilities in each group of breeds.
\end{abstract}

Keywords: breed, breed standard, dog, ethics, selection.

L'origine des chiens de race est réputée polyphylétique, et beaucoup de croisements se sont produits entre différentes lignées dont quatre d'entre elles, chacune reliée au loup de façon indépendante, ont constitué, sur des critères morphologiques, des pôles de regroupements inégaux (Parker et al. 2004 ; Wang \& Tedford, 2008) : quatre ensembles génétiques (A, B, C, D) rassemblent des populations d'origines géographiques identiques (Asie, Afrique), chacune étant composée de sujets aux morphologies semblables, ainsi que deux ensembles associés (E, F) et un plus récent $(\mathrm{G})$, européen, composé de 3 groupes $(\mathrm{a}, \mathrm{b}, \mathrm{c})$ (Figure 1). Le plus important en effectif et en diversité génétique est le groupe A (chiens primitifs, asiatiques et certains européens); il est admis que des croisements entre chien et loup, et/ou une domestication renouvelée de certains loups auraient eu lieu dans ces populations (Wayne \&Vilà, 2001 ; Lignereux, 2006).

1- Docteur vétérinaire, spécialiste en ophtalmologie. Courrriel : gilles.chaudieu@gmail.com 


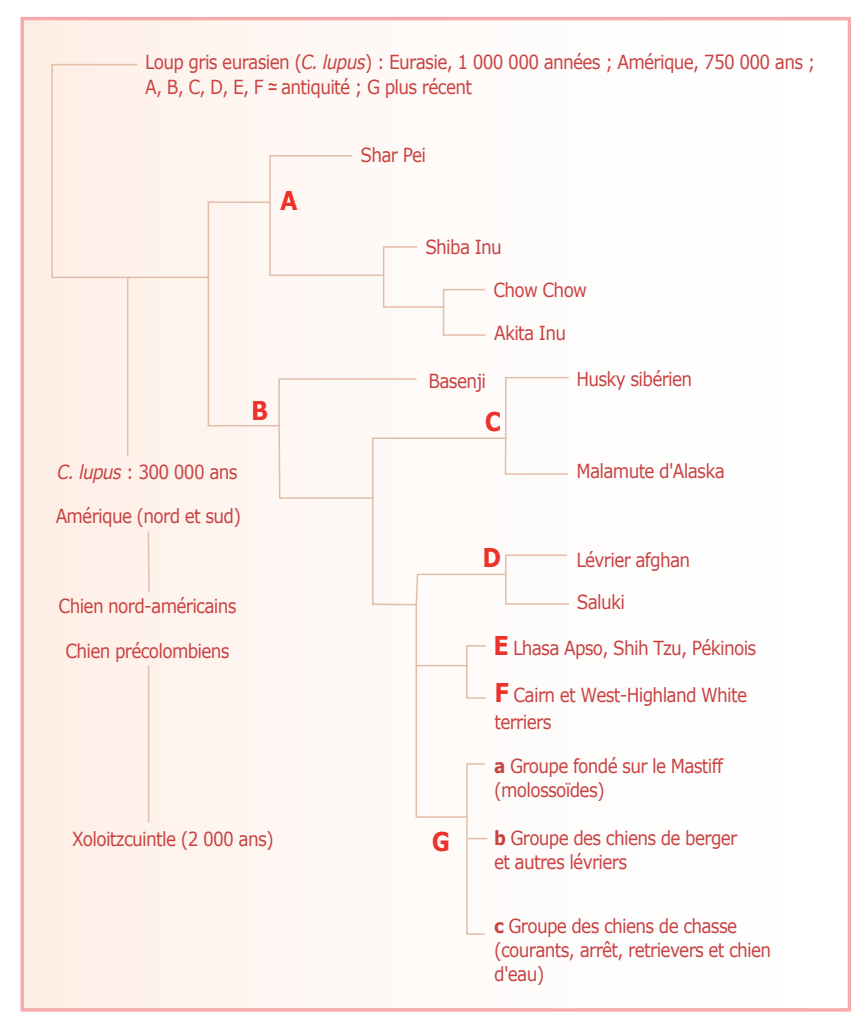

Figure 1. Raciation du chien : 4 ensembles génétiques (A, B, C, D : origines géographiques identiques - Afrique, Asie -, morphologies et fonctions comparables), 2 ensembles associés (E, F) et 1 plus récent (G, composé de 3 groupes $a, b$, c) (modifié d'après Parker et al. 2004).

\section{DU MORPHOTYPE ANCESTRAL CANIN AUX RACES PRIMAIRES}

L'Homme a façonné l'évolution du chien par la domestication de loups commensaux, dont sont issus les morphotypes utilitaires ancestraux. Géographiquement fixés, leurs performances ont été entretenues et progressivement améliorées en fonction des besoins humains. Cette évolution s'est faite de proche en proche dans des milieux hétérogènes, la différenciation du chien primitif polyvalent daterait du néolithique (10 000 ans avant notre ère), sans pression sélective humaine, avec passage progressif du statut de commensal d'opportunité à celui d'auxiliaire de l'Homme, au gré d'intérêts complémentaires des deux espèces (Denis, 2006 ; Licari, 2006 ; Licari 2021). Le morphotype primitif médioligne et mésocéphale de sujets de taille moyenne, à oreilles dressées, à la fois rapides et endurants, se distingue de celui du loup par des réductions du format, de la surface des attaches musculaires osseuses, de la largeur des arcades zygomatiques, de la crête occipitale et par un raccourcissement de la face, qui traduisent une diminution de la pression de sélection naturelle faite sur l'adaptation à la prédation, sans qu'on ne puisse l'interpréter comme une tentative de sélection intentionnelle par l'Homme (Olsen, 1985 ; Wayne, 2001 ; Wayne \& Vilà, 2001 ; Lignereux Y, 2006). Des représentations rupestres de morphotypes ancestraux à la poursuite du gibier et en action de conduite de troupeaux, la présence de chiens dans des sépultures humaines (Tchernov \& Valla, 1997, Licari, 2006), laissent à penser que le statut social et cynégétique du chien pourrait attester d'un début de sélection intentionnelle existant dès le septième millénaire avant notre ère.

Le chien non spécialisé n'est pas tant le résultat d'une sélection utilitariste plus ou moins planifiée que celui d'un processus multimillénaire conjoncturel d'apprivoisement, géographiquement dispersé, en foyers plus ou moins distants, où le commensalisme entre les deux espèces était la règle avant que ne soient clairement identifiables les fonctions attribuées au chien dans le cadre d'un processus de domestication : chasse interspécifique, protection des personnes et des biens, conduite des troupeaux (Wayne, 2001 ; Digard, 2006). La variabilité génétique de l'espèce canine, la plus importante qui se puisse rencontrer parmi les espèces domestiques (André \& Galibert, 2006), pourrait témoigner, selon certains auteurs, d'une cohabitation établie de l'Homme et du chien antérieure aux 15000 années de la domestication de ce dernier (Vilà et al. 1997 ; Lignereux, 2006) ; l'association d'ossements et d'empreintes de loups à des vestiges de présence humaine témoignent d'une possible " cohabitation prédomesticatoire " (Lignereux, 2006) plus de 30000 ans avant notre ère (Clottes J, 2001). Des morphotypes spécialisés émergeant à la faveur de conditions localement favorables ont pu induire les premières pressions sélectives utilitaristes, corollaires d'usages spécifiques du chien, la spécialisation fonctionnelle entraînant une adaptation morphologique consécutive :

- la chasse, avec le type graïoide (lévrier) de chasse à vue développé dans des régions à végétalisation peu dense et de faible hauteur : Sahara algérien et libyen, Égypte prédynastique et Mésopotamie, respectivement cinq et quatre siècles avant notre ère (Licari, 2006) ;

- la garde, avec le type molossoïde de protection des troupeaux, puissant et dissuasif, neuf siècles avant notre ère en Asie occidentale et en Afrique du nord-est, premiers territoires de domestication du bétail (Licari, 2006);

- le portage ou le traînage en Amérique et Europe du nord, en Sibérie, avec les chiens nordiques dont l'apparition ne peut pas être antérieure au quatrième millénaire avant notre ère pour des raisons de taille suffisante (Digard, 2006), sélectionnés sur leur résistance physique au trot sur de longues distances, chez qui des épisodes récurrents d'hybridation avec le loup ont été décrits (Clutton-Brock, 1995 ;Vila et al. 1997 ; Wayne et Vilà, 2001).

À partir de noyaux domestiqués fixés géographiquement se sont ainsi créées des populations aux caractères identifiables, qui ont constitué les races primaires, au sein desquelles des races secondaires, ancêtres de nos races actuelles, se sont spécifiquement développées : elles sont à la fois le résultat de contraintes environnementales locales imposées, et de croisements « améliorateurs " entre différents types de chiens les plus aptes à leurs fonctions à la faveur des migrations humaines d'abord, des possibilités de communications plus organisées entre les populations ensuite (Denis a, 2006). La classification des souches fondatrices des races actuelles en braccoïdes, lipoïdes, graïodes et molossoïdes (Mégnin, 1896) présente donc non seulement un intérêt morphologique, mais témoigne aussi d'une compréhension phylogénétique de la création des races canines. 
L'apparition de nouveaux types s'est faite peu à peu : bassets, chiens de chasse, petits chiens de compagnie, terriers... alors que la polyvalence du morphotype primitif continuait de s'incarner dans les races bergères, avec des différences de format, de longueur et texture de poil permises par la variabilité de l'espèce.

\section{DES RACES SECONDAIRES AUX STANDARDS DE RACES}

Par le choix et l'utilisation de certains sujets aux aptitudes remarquables en vue de conserver et améliorer éventuellement une population donnée, bien avant que la sélection au sens moderne du terme, ne se mette en place au XIX ${ }^{\text {ème }}$ siècle pour devenir la règle, nous avons vu qu'une sélection utilitariste avait amorcé le processus de raciation du chien. Les races actuelles ont finalement été créées en exploitant les possibilités génétiques de variation de l'espèce (continue, discontinue) par la retrempe et le métissage (Denis b, 2007). Définitivement, le chien « vit dans et près de la maison mais est élevé pour répondre à des besoins humains spécifiques "(Hemmer, 1990), les races constituant des isolats génétiques (André \& Galibert, 2006) dont la structure microsatellitaire serait identifiante avec une probabilité de $99 \%$ (Lignereux, 2006). Le premier standard, celui du Bulldog anglais, date de 1876 ; la création des livres généalogiques a ouvert la voie de la sélection " moderne " il y a 150 ans, formalisant une mainmise récente de l'Homme sur l'évolution des races canines par la sélection directionnelle de celles-ci.

\section{MOYENS ET BUTS DE LA SÉLECTION}

La sélection exploite uniquement la valeur génétique additive, ou variance génétique additive, qui correspond à la valeur d'élevage (breeding value) ; celle-ci mesure le potentiel génétique du reproducteur déterminé par les effets additifs de ses gènes (Denis b, 2007). Son but est de procurer le meilleur choix de reproducteurs en vue de conserver et améliorer une population donnée, en tendant vers un phénotype de beauté mais aussi de caractère idéalement représentatif de la race ; cette dernière notion est importante car elle témoigne de la fiabilité du chien dans l'exécution d'une fonction : à la fois "inféodé " à l'Homme et " autonome " dans son action, le chien est un mélange harmonieux de "soumission dans l'action et de justesse à la réponse à l'ordre " (Courreau, 2006). Toutefois, un siècle et demi de sélection telle que pratiquée doit nous faire prendre conscience que son but ne peut plus être uniquement de " formater " le chien de race pour servir les seules fins que des cynophiles auraient fixées. Des millénaires de cohabitation, domestication, de vie commune et d'intérêts partagés dont nous avons tenté de résumer les principales étapes, ont non seulement renforcé les capacités de l'espèce canine à s'adapter à l'Homme, mais aussi à exister pour elle-même dans cette situation de dépendance réciproque des deux espèces. L'éthique de sélection nous impose de protéger et promouvoir cette part d'action " autonome " dans les fonctions interspécifiques du chien, si nous voulons nous prémunir de certains excès présents dans des conceptions radicales actuelles des droits de l'animal (Donaldson \& Kymlicka, 2016).

\section{ÉTHIQUE VÉTÉRINAIRE ET SÉLECTION DES RACES CANINES}

En France, le Ministère de l'agriculture a délégué à la Société centrale canine (SCC) la tenue du livre généalogique de l'espèce canine, le LOF (Livre des origines français). Les Clubs de races, sous la responsabilité de leur organisme fédéral de tutelle (SCC), déploient sur le terrain leur politique de sélection, encadrant le travail des éleveurs.

La sélection canine est individuelle : elle résulte d'appréciations phénotypiques en classes homogènes, et se concrétise par les jugements, dont la répétition évalue la « valeur génétique » d'un sujet. Cette sélection diffère de celle des animaux de rente (usage d'indices de sélection) et est surtout généalogique, fondée sur les performances des ascendants et la constitution de pedigrees ; elle est aussi en partie effectuée sur la descendance au moyen de grilles de sélection, qui prend en compte la qualité des sujets issus d'un reproducteur pour son classement élite B, et l'associe à celle de son phénotype pour le classement élite A. Tous les acteurs doivent inscrire leurs interventions dans le sens d'un bénéfice pour le chien, notamment dans l'épanouissement des qualités de la relation Homme/animal, c'est-à-dire élever ce qu'il est convenu d'appeler de " bons chiens »; ils doivent aussi a minima respecter un primum non nocere dans l'évolution des phénotypes raciaux, c'est-à-dire élever de " beaux chiens » en bonne santé. La singularité et l'ancienneté des liens entre le chien et l'Homme imposent à ce dernier un " engagement moral ", qui passe par le respect de règles de "bonne conduite " dans la gestion des effectifs raciaux.

La légitimité du vétérinaire pour contribuer à la mise en place d'une politique de sélection équilibrée, comprise et acceptée par les éleveurs, Clubs de races et... les propriétaires, est évidente : il a compétence pour veiller à la santé génétique des effectifs et l'évolution raisonnable d'un type sans sacrifier les aptitudes naturelles de la race. La contribution vétérinaire est scientifique et technique ("cynotechnique »), ce qui ne doit pas la dispenser d'être, au bon sens du terme, cynophile : l'auteur argumentera cette opinion à la lumière de l'expérience acquise dans une race dont il est amateur et possesseur depuis 47 ans : le Colley (ou Collie) à poil long, et dans laquelle il a examiné de nombreux chiens en consultation ophtalmologique pendant une période équivalente.

\section{Le standard de race et la sélection phénotypique}

Par décret 69-667 du 14.06.1969, le standard est défini en France comme l'ensemble de " caractères héréditaires communs, avec indication de leur intensité moyenne d'expression ». Cette définition est heureusement prudente. D'autres le sont moins : "Le standard, c'est la matrice idéale de la race. " (Triquet, 1999, citant Queinnec) ; "Le texte [du standard] doit refléter l'image idéale et le véritable type de la race. " (Introduction au modèle du standard par la Fédération Cynologique Internationale, FCI, en 2009). Le standard type (modèle de Jérusalem, 1987) recommande des dessins (pas une photographie) ajoutés au texte parce qu'il n'y a pas de chien parfait, que la photographie représente le chien à un moment donné (le standard peut évoluer), que la 
morphologie du chien doit être en rapport avec une fonction et que la vision du chien par le juge doit être dynamique (Triquet, 2017). À la première page d'un standard FCI, sous ce dessin, figure la mention : «Illustration ne représentant pas forcément un exemple idéal de la race "... L'argumentation ne semble pas très cohérente... En France, les photographies des chiens « en statique " (parfois sur un podium d'exposition) occupent par ailleurs une partie conséquente des revues de clubs; elles ne reflètent que "l'idéal phénotypique du moment ", qui n'est parfois pas l'exacte expression du standard.

Celui-ci met en exergue des caractères quantitatifs fortement héritables et facilement modifiables compte-tenu de la plasticité physique de l'espèce : c'est avant tout un document morphologique et phanéroptique, parfois rédigé avec un soin qui confine à l'excès de détails (standards italiens), parfois de manière floue, imprécise, demandant un commentaire des juges "qui peut devenir un standard bis tendant à créer un chien bis " pour les standards britanniques (Triquet, 1999) (Encadré 1). Le juge doit faire preuve d'une solide connaissance de la race ou des races qu'il examine pour ne pas oublier le type racial moyen au profit d'une recherche de détails sans grande importance, ou en privilégiant un type morphologique qui s'écarte trop du type moyen (le " chien bis ").

\section{Quelques exemples}

- Standards britanniques : imprécis, rédigés peu scientifiquement et parfois maladroitement, flous, ils entraînent une présentation artificielle, une séparation fréquente des aptitudes et de la beauté, des possibilités multiples de titre... et de " chien bis ";

certains des changements apportés en 1986 ne sont ni respectés par les éleveurs, ni par les juges (le Bulldog devrait avoir la face moins courte !) ;

le standard est " un coup d'œil, une impression "; par exemple, l'oreille est attachée haute chez certains terriers mais le standard précise seulement le port ou l'écartement des oreilles, pas la hauteur d'attache : " assez haute » chez le Welsh terrier, " trop haute » est un défaut chez l'Airedale terrier...

Standards italiens : très détaillés, usant de termes savants, avec des rapports et des proportions (11 chez le Lagotto romagnolo, dont « le cou doit représenter les $8 / 5$ de la tête "!

- Standards espagnols : sur le modèle italien, avec moins de rigueur et de termes savants.

- Standards allemands et français : intermédiaires mais non exempts de jargon.

-Standards russes : langue claire, rédigés par des zootechniciens avec des paragraphes "type et constitution ", " particularités du comportement " et des indices de format (longueur du corps/taille), de hauteur du membre antérieur (distance olécrâne-sol/hauteur au garrot).

- Standards américains : "à l'anglaise » mais plus fouillés et mieux écrits.

\section{Important :}

- un standard flou peut conduire à créer des modèles au sein d'une race : modèle de beauté, modèle de travail, dont les Anglais sont les « inventeurs ";

- un standard trop fouillé peut devenir un carcan et figer la race sans lui permettre d'évoluer;

- Les considérations "pseudo-historiques» ou géographiques devraient être considérées comme hors-sujet dans un standard.

Encadré 1 : Des difficultés de l'interprétation des standards, selon $R$. Triquet (Dictionnaire encyclopédique des termes canins. L'Isle en Dodon: Maradi ; 1999, pp 531-4).

Deux races anglaises, le Foxhound et le Harrier, chiens courants sous responsabilité de l'association de vénerie, n'ont pas de standard, ce qui confirme si besoin était la séparation nette entre travail et beauté au Royaume-Uni (Triquet, 1999). Dans le modèle de Jérusalem de 2021 (traduit de l'anglais par R. Triquet), le standard du Collie à poil long, rough Collie (standard FCI n¹56) indique qu'il est un chien de berger... sans épreuve de travail, présenté comme " plutôt la version raffinée du chien de travail d'origine appartenant au berger écossais "; il est précisé que « beaucoup de ces sujets peuvent encore travailler s'ils en ont l'opportunité " et que "le Collie demeure un chien de travail ». S'il fallait encore s'en convaincre, travail et beauté sont considérés comme peu compatibles ! Le tempérament du Collie à poil long est décrit comme «aimable, joyeux et actif, ayant un bon comportement envers les enfants et autres chiens" : cette annexe rédactionnelle est typique de la sélection de beauté, devenue la règle exclusive dans la plupart des races, dont les standards ont éludé ou réduisent à une portion congrue les fonctions du chien telles qu'elles ont orienté son évolution phylogénétique jusqu'à la fin du XIX ${ }^{\text {ème }}$ siècle. Dans ces conditions, le vétérinaire doit rappeler qu'une mise en application éthique de ces derniers doit non seulement veiller à éviter une évolution irréversible du type sous l'effet de jugements liés à des interprétations déviantes du standard, mais aussi défendre l'« utilisation du chien, liée à des comportements, adaptés à une fin " (Courreau, 2017).

\section{Sélection et santé génétique}

"Toute race doit demeurer capable, par ses propres moyens, d'assumer une réorientation de sa sélection, c'est-à-dire d'évoluer vers un type morphologique ou des aptitudes un peu différents " (Denis, 2007). En 2020, pour 803 chiens inscrits au LOF, seulement 139 Collies ont été confirmés (https://www.centrale-canine.fr, Statistiques SCC), c'est-à-dire sont susceptibles de renouveler la population reproductrice de la race en passant par une grille de sélection à 6 niveaux, qui éliminera un nombre significatif d'entre eux de la cotation 1 à la cotation 6. Plus le pool de reproducteurs est faible, plus la pression de sélection phénotypique fondée sur l'ascendance avec transmission espérée des caractères, de fait associée à une pression consanguine, risque de s'exercer au détriment de la 
santé des chiens, simplement en respectant le standard : pénaliser de " grandes taches noires dans le poil de couverture ou le sous-poil " revient à sélectionner sur une extension de la panachure blanche. Pénaliser le ladre et l'œil «vairon ou taché de bleu " du Collie zibeline ou tricolore, l'un ou/et l'autre pouvant s'observer avec des panachures surtout si elles sont envahissantes (Denis, 2007), manque alors pour le moins de cohérence. Refuser "l'iris jaune-clair » du Collie "zibeline " est du même ordre dans la mesure où cette couleur témoigne de l'expression de l'allèle le moins dominant (iry) présent au locus Ir et soumis comme ses homologues $\left(\mathrm{Ir}^{+}, \mathrm{ir}^{\mathrm{m}}\right)$ à l'intervention de gènes modificateurs (Denis b, 2007). Tous ces "détails " induisent inutilement une pression sélective injustifiée et son corollaire, la pression consanguine.

La confirmation, spécificité française, vaut autorisation à reproduction, en position de cotation 1 de la grille de sélection, goulot d'étranglement progressif du nombre des reproducteurs qui seront sollicités jusqu'au niveau 6. Corpus de règles plus ou moins rigides de sélection phénotypique, le standard dépend du pays d'origine pour apporter des modifications à sa rédaction. Les points pénalisants ou éliminatoires sont, comme nous venons de le voir, souvent discutables. L'urgence à sensibiliser les clubs de races aux risques d'affaiblissement des bases génétiques raciales par pression sélective et usage de la consanguinité excessifs n'a pas échappé à la FCI : en 2010, une directive a invité à limiter le nombre de saillies des mâles reproducteurs (limite à $5 \%$ des chiots produits dans une race sur 5 ans par mâle reproducteur) ; la SCC permet à chacun de consulter les coefficients de consanguinité des reproducteurs utilisés sur LOF Select, et c'est une bonne chose. À terme, la dérive génétique, responsable d'un déficit de robustesse et de prolificité des sujets, peut s'installer et imposer la retrempe pour sauver une race devenue incapable d'assurer seule sa pérennité. Entre des sujets dont le coefficient de consanguinité est inférieur à $6,25 \%$ et ceux où il est supérieur à $12,5 \%$, existe une diminution moyenne de longévité d'une année (Leroy et al. 2014).

Dans un effectif donné, la fluctuation génétique des allèles prédispose à la "sortie " de maladies génétiques d'expression récessive (Charlet, 2004), d'autant plus que les pressions de sélection et de consanguinité ont contribué à limiter sa variabilité génétique (Oberbauer \& Sampson, 2001), imposant... une pression de sélection supplémentaire si un processus de dépistage/éradication des maladies est mis en place. Chez 71 chiots apparentés examinés dans des portées de 4 chiots en moyenne pour dépister l'anomalie de l'œil du Colley (AOC), 50 sujets (soit 70,4\%) étaient affectés d'hypoplasie/dysplasie chorö̈dienne (Figure 2), alors que l'estimation dans la population française avoisinait 41\% (Mathieu, 2004). La mutation autosomique récessive NHEJ1 (Non-Homologous End-Joining 1) identifiée comme responsable (Lowe et al. 2003) a permis la commercialisation d'un test de dépistage fiable largement utilisé. Cependant, l'affection, concomitante avec un défaut de vitalité des portées dans certains effectifs (Wallin Hakanson et al. 2000), a été rapportée comme non compatible avec les résultats du test génétique (Wallin Hakanson et al. 2000 ; Fredholm et al. 2016) dans certains effectifs, comme nous avons pu également le constater (Figure 3), d'où l'importance de l'examen vétérinaire clinique du fond d'œil.

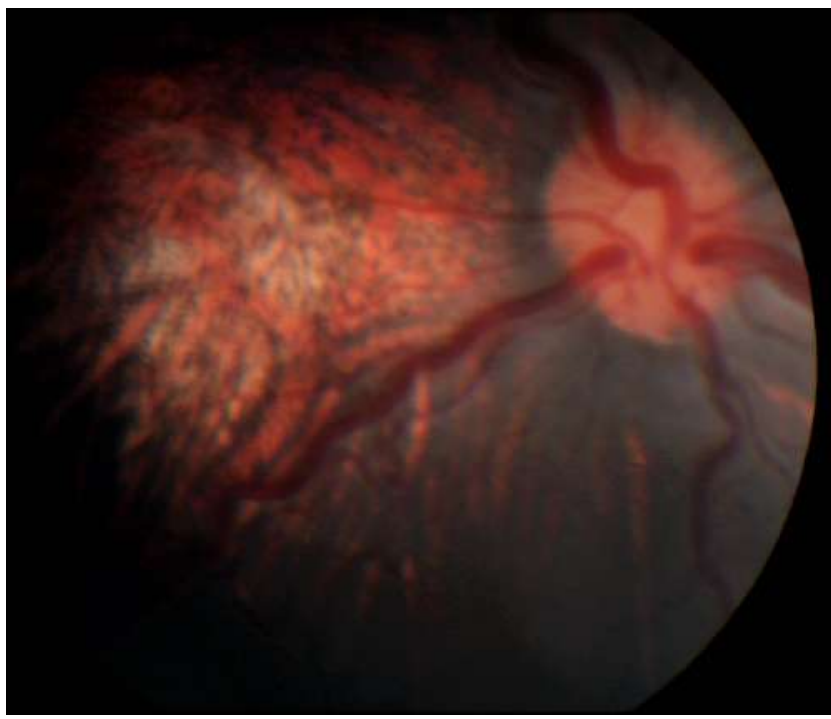

Figure 2 : Fond d'œil d'un Collie à poil long montrant une hypoplasie/dysplasie choroüdienne (œil droit). Noter l'absence de pigmentation choroïdienne (sclère visible); l'hypoplasie, l'irrégularité de calibre et l'anastomose des vaisseaux choroïdiens temporalement à la papille optique.

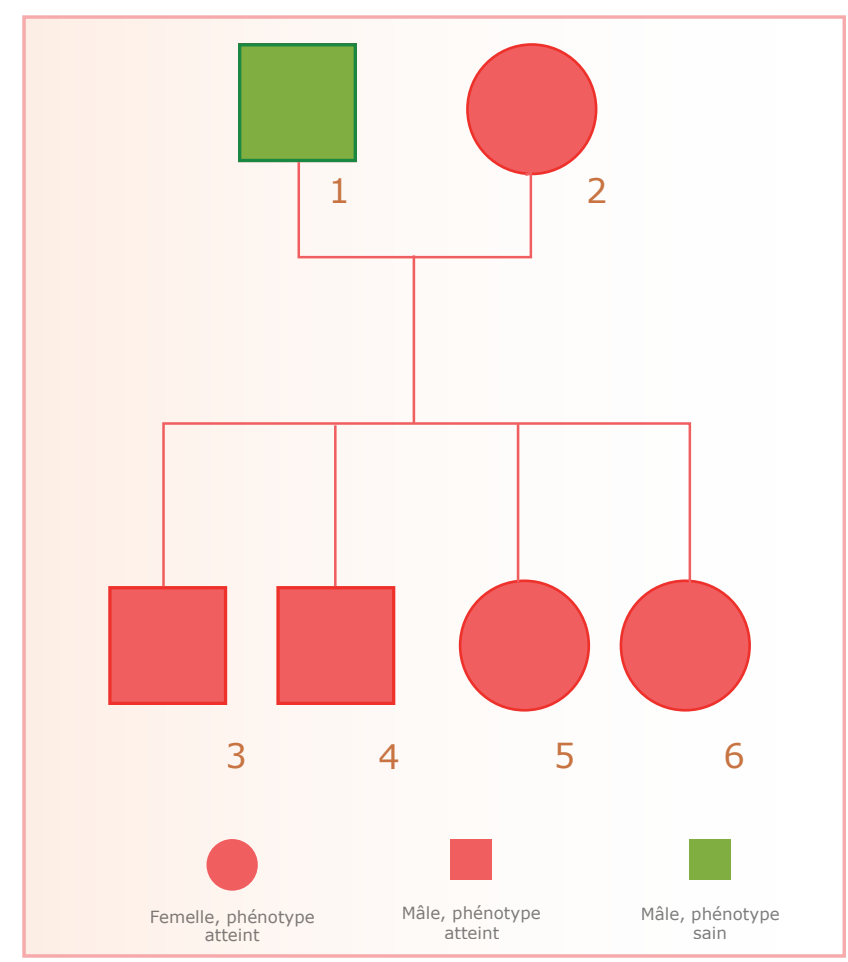

Figure 3 : Transmission de l'hypoplasie/dysplasie choroïdienne dans une portée de 4 chiots Collie à poil long. Les chiens 1 et 2 ont été examinés à 7 semaines puis à 2 ans d'âge; chiots 3, 4, 5 et 6 examinés à 7 semaines d'âge. Le statut de la portée est incompatible avec un mode de transmission autosomique récessif. 


\section{Sélection, bien-être et aptitudes}

Dans le standard FCI du Collie à poil long, on peut lire : «Tout écart par rapport à ce qui précède [le standard] doit être considéré comme un défaut qui sera pénalisé en fonction de sa gravité et de ses conséquences sur la santé et le bien-être du chien et sur sa capacité à accomplir son travail traditionnel ». Or, en France comme au Royaume-Uni, les "types idéaux " de beauté ne travaillent plus, les types de travail ne sont plus dans le standard... et ainsi le beau s'oppose artificiellement au bon ! Le Collie à poil long de beauté s'est ainsi progressivement séparé du Scotch Collie ancestral dans la deuxième moitié du XIX ${ }^{\text {ème }}$ siècle ; il est rapporté que la reine Victoria, ayant acquis son premier Collie en 1846, aurait activement contribué à la création de "show dogs " par métissage avec le lévrier Barzoï (What did Collies originally look ? https://www.oldtimefar mshepherd.org)! Le Scotch Collie à poil long, originaire du nord des Highlands, souvent porteur de double ergot postérieur comme un certain nombre d'autres vieilles races bergères, est maintenant dénommé "Farm Collie ", que quelques passionnés de la race élèvent et utilisent encore, bien que l'effectif ne soit pas reconnu comme race par la FCI
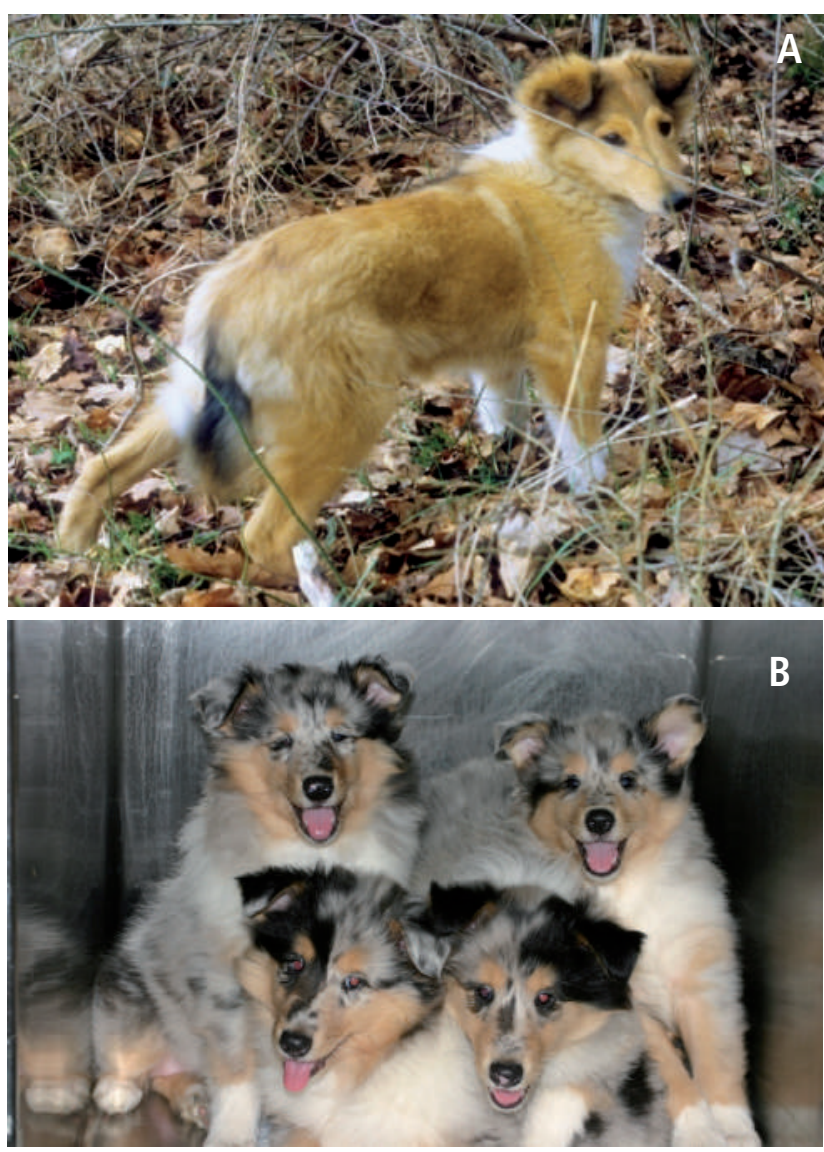

Figure 4. La qualification "Farm Collie » s'applique à tout chien dont le type correspond à celui du Scotch Collie ancestral et/ou qui travaille au troupeau. A. "Farm Collie » âgé de 2 mois (chien français, non LOF, 1980).

B. Portée de Collies à poil long bleu-merle de beauté, âgés de 7 semaines.
Dans la mesure où « l'excellence des qualités intérieures du chien qui peuvent lui attirer les regards de l'Homme " (Buffon, 1839) représente le moteur de la passion cynophile, la plus sûre et la meilleure définition d'un standard de race est bien la " description méthodique de l'archétype racial» (Denis, 2007), et une véritable iconographie jointe à son texte (avec le chien aux allures dans son milieu naturel) serait souhaitable, dans la mesure où santé et morphologie des chiens devraient être liées. La morphologie n'est qu'un critère de classification raciale (parfois trompeur), où doivent aussi compter les origines, et l'incitation à une utilisation conforme au potentiel racial qui devrait à ce titre figurer explicitement dans les standards. Par l'édition de documents généalogiques enrichis des résultats en concours de beauté, aux tests de travail et de santé, consultables par tous sur LOF Select (https://wwwcentrale-canine.fr), la SCC a beaucoup travaillé pour répondre maintenant à ces exigences. Les critères de beauté valident majoritairement la " réussite d'une sélection ", à l'exception notable du Border Collie "de troupeau " dont la confirmation est soumise à une épreuve de travail ; l'avantage accordé par les jugements à un certain type racial peut être déterminant, voire excessif... jusqu'à substituer un hypertype à l'archétype racial du standard : la formation initiale et continue des juges doit mettre l'accent sur ce risque et se structurer autour d'une culture cynotechnique générale approfondie susceptible de donner les bases d'un recul nécessaire par rapport à l'inévitable " entre-soi " des stages pratiques. Prenons l'exemple des chiens brachycéphales, qui alimentent actuellement le débat sur le respect du bien-être animal : sachant que l'indice crânio-facial (ICF) considéré comme idéal est compris entre 0,3 et 0,5 pour s'affranchir au mieux du risque de syndrome obstructif respiratoire (Michel, 2017), mais qu'il serait injuste de jeter l'anathème sur certaines races produites par sélection avec un ICF voisin de 0,2, parfois inférieur (Packer et al. 2015 ; Guintard et al. 2020), quelle doit être l'attitude la plus conforme au respect de l'éthique vétérinaire sur ce point? D'abord, il faut produire un état des lieux en évaluant les valeurs moyennes de l'ICF dans les races concernées (Tableau 1) : des données existent (Guillon et al. 2016), il convient de les confronter à la fréquence avec laquelle une détresse respiratoire mais aussi d'autres affections liées au morphotype (cutanées, oculaires) sont rapportées dans ces mêmes races (Cazorla, 2018). Parallèlement, des critères d'appréciation objectifs simples (Tableau 2, Figures 5A et 5B, 6 et 7), dont la mise en ouvre par les vétérinaires et la prise en compte par les éleveurs sont claires et facilement applicables, devraient contribuer à une sélection de reproducteurs et une production de chiots compatibles avec une meilleure prise en compte du bien-être et de la santé des chiens brachycéphales. Le test d'effort, mis en place par un certain nombre de clubs concernés, en est un bon exemple. 


\begin{tabular}{|l|l|l|l|l|}
\hline Race & $\begin{array}{l}\text { Packer RMA et } \\
\text { al. (2015) : chiens } \\
\text { référés de 12/2010 } \\
\text { à 01/2012 }\end{array}$ & $\begin{array}{l}\text { Packer RMA et al. } \\
(\mathbf{2 0 1 5}): \text { chiens non } \\
\text { référés de 07/2012 } \\
\text { à 04/2013 }\end{array}$ & $\begin{array}{l}\text { Guintard et al. } \\
\mathbf{2 0 2 1} \text { : chiens } \\
\text { examinés en 2020 }\end{array}$ & $\begin{array}{l}\text { ICF en 1910 } \\
\text { (Guintard } \text { et al. } \\
\mathbf{2 0 2 1})\end{array}$ \\
\hline Affenpinscher & $0,20(1)$ & $0,23(31)$ & $0,25(2)$ & 0,25 \\
\hline Carlin & $0,08(32)$ & $0,12(32)$ & $0,08(3)$ & 0,22 \\
\hline Bouledogue français & $0,19(3)$ & $0,18(4)$ & $0,16(10)$ & 0,20 \\
\hline Bulldog anglais & $0,22(16)$ & $0,25(6)$ & $0,21(6)$ & 0,25 \\
\hline Boston terrier & $0,14(6)$ & $0,23(2)$ & $0,18(7)$ & 0,24 \\
\hline Dogue de Bordeaux & $0,36(6)$ & $0,36(6)$ & $0,35(3)$ & 0,38 \\
\hline Griffon bruxellois & $0,13(2)$ & $0,15(20)$ & $0,16(5)$ & 0,22 \\
\hline Pékinois & $0,12(3)$ & $0,11(3)$ & $0,10(3)$ & 0,25 \\
\hline Shih Tzu & $0,20(13)$ & $0,22(7)$ & $0,18(4)$ & 0,23 \\
\hline
\end{tabular}

Tableau 1 : Valeurs moyennes comparées de l'ICF* dans quelques races brachycéphales: aucune des races n'a d'ICF $>0,3$; certaines ont un ICF stable ou peu diminué en un siècle (Affenpinscher, Bouledogue français, Bulldog anglais, Dogue de Bordeaux, Shih Tzu); d'autres ont subi une diminution d'ICF impressionnante (45 à $64 \%$ pour le Carlin, 52 à $60 \%$ pour le Pékinois); certaines enfin présentent une diminution d'ICF variable selon les sujets ( 7 à $42 \%$ pour le Boston terrier, 27 à $41 \%$ pour le Griffon bruxellois).

*ICF, ou ratio crânio-facial, ou CFR (cranio-facial ratio) : rapport entre la distance comprise entre la suture fronto-nasale et l'extrémité rostrale de l'os nasal et celle comprise entre la protubérance occipitale externe et la suture fronto-nasale.

\begin{tabular}{|c|c|c|}
\hline Critères de jugement & À contrôler & Conditions de mise en ouvre \\
\hline $\begin{array}{l}\text { Bruits respiratoires } \\
\text { anormaux }\end{array}$ & $\begin{array}{l}\text { Au repos, le chien éveillé renifle, ronfle } \\
\text { ou émet un stridor respiratoire }\end{array}$ & $\begin{array}{l}\text { Évaluation des chiens au repos et à l'exerc- } \\
\text { ice par un vétérinaire lors d'un test d'effort }\end{array}$ \\
\hline Ouverture des narines & $\begin{array}{l}\text { Sténose modérée } \\
\text { Sténose sévère }\end{array}$ & Examen vétérinaire clinique des narines \\
\hline Indice crânio-facial & $\begin{array}{l}0,3<\mathrm{ICF}<0,5 \\
\mathrm{ICF} \leq 0,3\end{array}$ & Mesure de l'ICF si besoin \\
\hline Pli nasal & $\begin{array}{l}\text { Pli nasal sans contact de poils avec la } \\
\text { cornée; sans humidité (absence de } \\
\text { macération dans le pli) } \\
\text { Pli nasal avec poil au contact de la cornée } \\
\text { ou de la conjonctive; humidité } \\
\text { (macération dans le pli) }\end{array}$ & Examen vétérinaire clinique du pli nasal \\
\hline Sclère & $\begin{array}{l}\text { Blanc de l'œil visible sur moins de } 2 \text { qua- } \\
\text { drants quand le chien regarde face à lui } \\
\text { Blanc de l'œil visible sur au moins } 2 \\
\text { quadrants quand le chien regarde face à } \\
\text { lui (orbite peu profonde et/ou fente } \\
\text { palpébrale trop grande) }\end{array}$ & $\begin{array}{l}\text { Examen vétérinaire clinique } \\
\text { Examen vétérinaire officiel par un spécialiste } \\
\text { en ophtalmologie vétérinaire ou un panellist } \\
\text { ECVO/AFEP MHOC }\end{array}$ \\
\hline Réflexe palpébral & $\begin{array}{l}\text { Fermeture complète des paupières } \\
\text { Fermeture incomplète des paupières }\end{array}$ & $\begin{array}{l}\text { Examen vétérinaire clinique } \\
\text { Examen vétérinaire officiel par un spécialiste } \\
\text { en ophtalmologie vétérinaire ou un panellist } \\
\text { ECVO/AFEP MHOC }\end{array}$ \\
\hline
\end{tabular}

Tableau 2: Critères de sélection de reproducteurs dans les races brachycéphales : toléré, à proscrire, à réaliser en pratique (modifié d'après " Proposals of the Duch Kennel club for the breeding of brachycephalic dogs »).

*ICF, ou ratio crânio-facial, ou CFR (cranio-facial ratio) : rapport entre la distance comprise entre la suture fronto-nasale et l'extrémité rostrale de l'os nasal et celle comprise entre la protubérance occipitale externe et la suture fronto-nasale. 


\section{CONCLUSION}

Ces quelques formules de Raymond Triquet, grand éleveur et ancien Président du Club des amis du Dogue de Bordeaux, auteur d'un dictionnaire encyclopédique des termes canins qui fait autorité, longtemps représentant de la France à la FCI, fournissent une trame conclusive adaptée au "balisage " d'une approche vétérinaire de la sélection en élevage canin conforme à l'éthique professionnelle. Voici ces propositions (Triquet b, 2017). " Pour lutter contre l'hypertype, supprimez-en la cause : supprimez les expositions ! " : les supprimer non, les modifier pourquoi pas, en mettant mieux en valeur les lots d'élevage et de reproducteurs, les résultats du Concours général agricole où chaque sujet distingué fait l'objet d'une évaluation cynotechnique par un vétérinaire ; "Le bulletin du Club est un élément important de la lutte contre l'hypertype en ne se contentant pas des photos du champion en statique " : faire du bulletin le support pédagogique privilégié pour la formation des adhérents, éleveurs ou amateurs de la race, publier régulièrement les "données santé ", montrer les chiens tels qu'il faudrait les voir contribueraient à la promotion de bonnes pratiques d'élevage et à l'éducation de tous, particuliers acquéreurs de chiens inclus ; "Le standard bien formulé et bien compris par les juges et les éleveurs, publié et republié par le Club de race, peut faire des miracles !": veiller à la formulation des standards (proportions, rapports, angles..., Encadré 1) et à la qualité de leurs traductions (notamment pour les pays non-membres de la FCI), structurer la formation initiale et continue des juges et des responsables de Clubs, sont des points essentiels afin de sélectionner des types raciaux où le respect de la santé du chien est la meilleure des garanties d'un développement harmonieux de ses aptitudes raciales.

L'auteur déclare ne pas avoir de conflits d'intérêts.

\section{BIBLIOGRAPHIE}

- André C \& Galibert F. La domestication du chien : apports de la génétique moléculaire : données bibliographiques. Ethnozootechnie $2006 ; 78: 29-32$.

- Buffon GLL. Histoire naturelle générale et particulière, avec la description du cabinet du Roy, 1749-1789. CEuvres complètes. Tome 5. Paris : Imprimerie royale ; 1839.

- Cazorla AM. Perception de la santé des chiens brachycéphales par leurs propriétaires : étude bibliographique et enquête auprès des propriétaires. Thèse de doctorat vétérinaire, Toulouse : université Paul Sabatier ; 2018, 199 p.

- Charlet K. Principales maladies héréditaires ou présumées héréditaires dans l'espèce canine. Prédispositions raciales. Thèse de doctorat vétérinaire, Maisons-Alfort : Faculté de Médecine de Créteil ; 2004, 267 p.

- Clutton-Brock J. Origins of the dog: domestication and early history. In: The domestic dog, its evolution, behaviour and interactions with people. Serpell JA, ed. Cambridge : Cambridge University Press ; 1995, pp 7-20.

- Clottes J. La grotte Chauvet. L'art des origines. Paris : Seuil ; 2001.

- Courreau J-F. Évolution de la sélection du chien sur ses fonctions utilitaires. Ethnozootechnie 2006 ; 78 : 205-10.

- Courreau J-F. Génétique et standard chez le chien. In : Standards, santé et génétique chez le chien. Guintard $\mathrm{Cl}$,
Leroy G, éds. Aubervillliers : SCC ; 2017, pp 137-52.

- Denis B. Domestication et raciation primaire : la démarche récurrente, à partir des races actuelles. Ethnozootechnie $2006 ; 78: 67-70$.

- Denis B. Autour de la notion de race. In : Génétique et sélection chez le chien. Seconde édition. Paris : PMCAC-SCC ; 2007, pp 231-42.

- Digard J-P. Essai d'ethno-archéologie du chien. Ethnozootechnie 2006 ; 78: 33-40.

- Donaldson S \& Kimlicka W. Zoopolis. Une théorie politique des droits des animaux (2011), traduit de l'anglais par Pierre Madelin. Paris: Alma éditeur ; 2016.

- Fredholm M, Larsen RC, Jönsson M, Söderlund MA, Hardon T, Proschowsky HF. Discrepancy in compliance between the clinical and genetic diagnosis of choroidal hypoplasia in Danish Rough Collies and Shetland Sheepdogs. Anim Gen 2016; 47: 250-2.

- Guillon M, Borvon A, Thorin C, Betti E, Olier A, Guintard C. Étude crâniométrique d'un échantillon de chiens de races variées. Bull Soc Sci Nat de l'Ouest de la France 2016 ; 38, 3 : 113-29.

- Guintard Cl, Denis H. Morphologie faciale des brachycéphales. Évolution depuis la fin du XIXe siècle et perspectives actuelles. Troisième parie : synthèse, bilan et perspectives. Centrale Canine Magazine ; 2020 ; 210 : 29-31.

- Leroy G, Phocas F, Hédan B, Verrier E,
Rognon X. Inbreeding impact on litter size and survival in selected canine breeds. Vet Journal 2014; 1: 74-8.

- Licari S. Éléments d'émergence des principaux types de chiens à la lumière de témoignages iconographiques. Ethnozootechnie 2006 ; 78 : 47-66.

- Licari S. La raciation canine. Première partie. Centrale canine magazine 2021; $211: 23-8$.

- Lignereux Y. Des origines du chien. Ethnozootechnie 2006; 78: 11-28.

- Lowe J, Kukekova A, Kirkness E, Langlois M, Aguirre GD, Acland GM et al. Linkage mapping of the primary disease locus for collie eye anomaly. Genomics 2003; 82: 86-95.

- Hemmer H. Domestication: the decline of environmental appreciation. Cambridge University Press; 1990.

- Mathieu A-S. Prévalence de l'anomalie de l'œil du Colley chez le colley à poil long : étude de 336 examens oculaires réalisés de 1992 à 2004. Thèse de doctorat vétérinaire, Lyon : Université Claude Bernard de Lyon 1; 2004, 80 p. - Mégnin P. Les races de chiens, histoire, origine, description. Vincennes : Bureau de l'éleveur ; 1896.

- Michel M. Les hypertypes chez les chiens et chats de race : étude bibliographique et observationnelle. Thèse de doctorat vétérinaire, Lyon : Université Claude Bernard de Lyon 1; 2017, 162 p. - Oberbauer M \& Sampson J. Pedigree 
analysis, genotype testing and genetic counselling. In: The genetics of the dog. Ruvinsky A \& Sampson J, eds. Wallingford: CABI publishing; 2001, pp 461-85.

- Olsen SJ. Origins of the domestic dog. The fossil record. Tucson: University of Arizona press; 1995, pp 118-120.

- Packer RMA, Hendricks A, Tivers MS, Burn CC. Impact of facial conformation on Canine Health: Brachycephalic Obstructive Airway Syndrome, PloS ONE, 2015,10(10): e0137496.doi : 10.137/journal.pone.013749.

- Parker HG, Kim LV, Sutter NB, Carlson S, Lorentzen TD, Malek TB et al. Genetic structure of the purebred domestic dog. Science 2004; 304: 1160-4.
- Tchernov E \& Valla FF. Two new dogs, and other Natufian dogs, from the southern levant. J Archeological Sci 1997; 24: 64-95.

- Triquet R. Dictionnaire encyclopédique des termes canins. L'Isle en Dodon: Maradi ; 1999, pp 531-4.

- Triquet R. La lutte contre les hypertypes. Le point de vue d'un vieux cynophile. In : Standards, santé et génétique chez le chien. Guintard $\mathrm{Cl}$, Leroy G, éds. Aubervilliers : SCC; 2017, pp 108-21.

- Vilà C, Salvolainen P, Maldonado JE, Amorim IR, Rice JE, Honeycutt RL et al. Multiple and ancient origins of the domestic dog. Science 1997; 276: 1687-9.

- Wayne RK. Consequences of domesti- cation: morphological diversity of the dog. In: The genetics of the dog. Ruvinsky A \& Sampson J, eds. Wallingford: CABI publishing; 2001, pp 43-60.

- Wayne RK \& Vilà C. Phylogeny and origin of the domestic dog. In: The genetics of the dog. Ruvinsky A \& Sampson J, eds. Wallingford: CABI publishing; 2001, pp 1-14.

- Wallin Hakanson B, Wallin Hakanson N, Hedhammar A. Collie eye anomaly in the rough Collie in Sweden: Genetic transmission and influence on offsprings vitality. J Small Anim Pract 2000; 41:254-8.

- Wang X \& Tedford RH. Dogs: Their Fossil Relatives and Evolutionary History. New York : Columbia University Press ; 2008. 